\title{
Severe burns with complications successfully managed in a non specialized unit
}

\author{
Perera R, Kawindi 1
}

Burns exert a catastrophic influence on people in terms of human life, suffering, disability, and financial loss. More than an estimated 2 million people in the United States experience burn injuries. 5053 deaths were reported in 1991. Burn injuries are extremely complex and elicit physiologic and metabolic interactions involving all major organ systems (1).

This case is important because this patient was able to ssurvive with flame burns of $75 \%$ of body surface area with the management in a ward setting and she developed some noteworthy complications.

\section{Case history}

A 33 year old patient was admitted to surgical casualty with extensive burn injury, which was estimated to be $75 \%$ of body surface area. She alleged that she was burnt by putting kerosene oil over the body. On admission she was breathless and was in severe pain. Pulse rate was $60 \mathrm{bpm}$ with weak pulse. Blood pressure was difficult to be measured. Initial resuscitation was done with attention to airway and breathing with Oxygen support. Fluid resuscitation was done according to Muir and Barclay formula in the first 36 hours and then adjusted according to the urine output. Intramuscular pethidine was used for initial pain management. Patient had daily baths followed by local application of silver sulphardiazine cream. Intravenous cefuroxime was started. A high protein diet was given. Physiotherapy was given for limbs. Patient was psychologically motivated with her mother's support as a bystander. She had recurrent infections of burn wounds which were dealt with appropriate microbiological assessment and relevant antibiotics. Wound excision was performed as required. She became anaemic in spite of repeated blood transfusions with close supervision by the transfusion specialist and the haematologist. A definite cause was not found to account for anaemia. So bone marrow suppression and nutritional deficiencies were postulated. Iron supplements and subcutaneous erythropoietin were helpful at this moment. Blood transfusions were

General Surgical Unit, District General Hospital Matara done to keep haemoglobin level around $8 \mathrm{~g} / \mathrm{dl}$. She became severely hypoalbuminaemic. High protein diet, albumin and fresh frozen plasma transfusions were given to counteract hypoproteinaemia but albumin level showed only a slight increase. Patient developed a leak of copious amount of fluid from one of her leg which was later managed as a lymphatic leak. Compression bandages and a skin graft settled it completely. She had disfiguration of both of her ear lobes which was planned to be attended later. Finally she was discharged from the hospital after a long stay of four months.

\section{Discussion}

Burn injury is a common cause of morbidity and mortality. The outcome of the treatment depends on patient's condition. More than 50\% Total Body Surface Area (TBSA) involvement is very critical and outcome is unpredictable. Burns with $>80 \%$ TBSA involvement are most of the time provided comfort care only. All burn patients should be evaluated first as trauma patient paying attention to airway, breathing and circulation. Total body surface area involved should be estimated correctly. This is best estimated using the Lund-Browder chart supplemented by the use of patient's palm to represent $0.5 \%$ of their body surface area (2).

The goal of fluid resuscitation in burn is to restore and preserve tissue perfusion in order to avoid ischemia (3). This can be done by using several formulas. Widely used formulas are Muir and Barclay formula and Parklands formula (4). Burns are very painful. Goal of burn pain management is to keep pain within acceptable levels. During acute phase of burn injury intravenous or subcutaneous opioids are used. Following the acute period oral analgesics and suppositories of opioids or NSAIDS can be used.

Hyper metabolism and protein hyper catabolism have been noted in burn patients. Aggressive nutritional support should be provided during acute phase of recovery (2). Enteral feeds are recommended over parenteral feeds. Oral or enteral feeding should be started preferably within 1st 24hours. A high protein diet such as a calorie nitrogen ratio of $110: 1$ is associated with fewer infections and better survival (2).

Anaemia is a pervasive and difficult to treat consequence of severe burn injury. Anaemia of burn patients can be divided in to acute blood loss anaemia and anaemia of critical illness. Acute blood loss anaemia occurs during 
first 2 weeks after burn injury. This is caused by direct blood loss from burn injury and surgical management of wounds. Anaemia of critical illness occurs after resolution of initial acute injury. This is due to inability to produce enough red blood cells to meet demand. It is a multifactorial entity related to variety of factors including wound care, phlebotomy, impaired nutrition and metabolism, blunted erythropoietin production and reprioritisation of bone marrow cell production(5). Transfusion of blood products are an immediate and effective treatment for anaemia. But it has several consequences such as infections and transfusion related acute lung injury. An increase in erythropoiesis with erythropoietin administration has a greater impact on restricting transfusion rates (6).

Currently mortality rates related to severe burn injuries continue to steadily decline due to the standardization of multidisciplinary approach. When considering wound management, properly washed burn wounds should be dressed with silver sulphadiazine. Surgery is planned 24-36 hours post burn and after 48 hours in major burn (7). Tangential excision and grafting depends on the availability of donor sites. As Sri Lanka doesn't have skin banking facilities severe burn patients are allowed to heal spontaneously. Psychological support is important for the patient and family members, explaining prolonged hospital stay and considerable morbidity and mortality.

\section{References}

1. Thermal Burns Author: Richard F Edlich A,; Chief Editor: Jorge I de la Torre

2. Practice guidelines for burn care.www.ameriburn. org/practiceguideline.2013.

3. Kasten KR, Makley AT, Kagan RJ. Update on the critical care management of severe burns,2011 Jul-August

4. Moore FD. The body weight budget:basic fluid therapy for the early burn

5. Robert IO. Burn resuscitation and early managementNov 24,2015

6. Walsh TS, Saleh EE.Anaemia during critical illness. $\mathrm{Br}$ J Anaesth.2006;97:278-91

7. Joseph A. Posluszy Prichard L. Gamelli.Anaemia of thermal injury: combined acute blood loss anaemia and anaemia of critical illness.www.ncbi.nlm.nih. gov.pubmed

8. Guidelines for the management of the patient with a burn injury-Collage of Surgeons Sri Lanka-2007 